\title{
OBSERVABLE EFFECTS OF ROTATION ON STELLAR ABUNDANCES
}

\author{
SUCHITRA BALACHANDRAN \\ Department of Physics and Astronomy, University of North Carolina, \\ CB \#3255 Phillips Hall, Chapel Hill, NC 27599-3255.
}

ABSTRACT This is a review of our understanding of the effects of rotation on the stellar interior through an examination of observed surface stellar abundances. First, the evolution of angular momentum from the pre-main sequence through the giant phase is summarized. Attempts are then made to decipher the signature of abundance anomalies due to meridional circulation and rotational braking with reference to available theoretical models. Finally, special cases such as tidally-locked binaries and chromospherically-active giants are examined to determine if their rotational peculiarites have induced abundance anomalies.

\section{INTRODUCTION}

This review is a testimony to the volume of work that has been carried out in this area in the last decade, to the sophistication of our current understanding of the stellar interior, and to the belief that we are now of the threshold of being able to perform even more detailed comparison between observations and models. Until the beginning of the last decade, our knowledge of rotational velocities in stars was limited by our inablity to obtain high resolution spectra of faint objects. We knew that early-type stars exhibited a spread in rotational velocities which remained largely unchanged from the age of the Pleiades to older stars in the field (Kraft 1970). Bright, low-mass stars in the field were seen to be spinning slowly and it was assumed that these had been rotating slowly all their lives.

Very little was known about the effects of rotation on stellar composition. Observations of field giants revealed that a large fraction had smaller ${ }^{12} \mathrm{C} /{ }^{13} \mathrm{C}$ ratios (see Lambert and Ries 1981 and references therein) i.e. greater mixing has occurred, than predicted by standard dredge up theory. Meridional circulation induced mixing was examined in an effort to explain this anomaly 
(Dearborn, Eggleton and Schramm 1976) and it was concluded that greater mixing was possible in the more massive stars. The large fraction of low ${ }^{12} \mathrm{C} /{ }^{13} \mathrm{C}$ ratio giants however indicated that it was the low-mass stars that were more extensively mixed (Scalo and Miller 1978). This debate could not be resolved since it is not possible to determine the masses of field redgiants with any accuracy and abundances in cluster giants could not be measured with existing spectrographs and detectors.

\section{EVOLUTION OF ANGULAR MOMENTUM}

The revolution in this field began with the discovery through photometry of rapidly rotating $\mathrm{K}$ dwarfs in the Pleiades cluster by van Leeuwen and Alphenaar (1982). Since those observations the database of rotational velocities in young clusters has increased by leaps and bounds. The large number of studies have resulted in an entire conference devoted to the subject (Angular Momentum Evolution of Young Stars, ed. Catalano and Stauffer, 1991) and the interested reader is referred to extensive reviews in that volume. Here I will merely touch upon key observations that reveal the status of our understanding on the subject.

\section{On the Main Sequence}

Once on the main sequence, stars of different spectral types spin-down at varying rates (see Stauffer 1991 for a review). Comparing the young clusters Alpha Persei (turn-off age $~ 0.05$ Gyr) and the Pleiades (turn-off age $\sim 0.07$ $\mathrm{Gyr}$ ), one sees rapidly rotating $\mathrm{G}$ and $\mathrm{K}$ dwarfs in the former (Stauffer, Hartmann and Jones 1985; 1989; Prosser 1992), but by the age of the Pleiades, the $\mathbf{G}$ dwarfs have spun down while rapidly-rotating $\mathbf{K}$ dwarfs are still observed (Stauffer and Hartmann, 1987). By the age of the Hyades (turn-off age $\sim 0.6 \mathrm{Gyr}$ ) both $\mathrm{G}$ and $\mathrm{K}$ dwarfs have spun down but some relatively rapidly-rotating $M$ dwarfs with velocities upto $20 \mathrm{~km} / \mathrm{s}$ are still observed (Stauffer, Hartmann and Latham 1987; Radick et al. 1987). This can be broadly understood if the envelope is assumed to decouple from the core so that the increasing fractional mass of the envelope from $G$ to $K$ to $M$ dwarfs results in the longer spin-down timescale. A rough estimate of this spin-down timescale for $G, K$ and $M$ dwarfs can be obtained from these three clusters: $G$ dwarfs spin-down in a 20-30 million years, $K$ dwarfs in 100 million years and $M$ dwarfs in a several hundred million years.

An interesting feature is the large fraction of slowly rotating stars in both young clusters. Stauffer et al. (1989) pointed out that since these are seen even among G dwarfs in the very young cluster IC 2391 (turn-off age $~ 0.02 \mathrm{Gyr}$ ) an age spread within the cluster may be ruled out as their cause. Further, the 
fraction of slow rotators is too large to be accounted for as a result of near-poleon orientations of the inclination angle. It hence appears that stars arrive on the main sequence with a genuine spread in rotational velocities. The simple spindown picture is complicated by a number of Pleiades $\mathrm{K}$ dwarfs seen to be rotating at smaller velocities ( $v$ sini 's) than comparable temperature stars in the Hyades. Since the Hyades velocites are derived from rotational periods (Radick et al. 1987), they are true velocites and not upper limits. Unless the stellar envelope recouples with the rapidly rotating core in the intervening period, these Pleiades K dwarfs will be spinning much more slowly at the age of the Hyades. There may be other cluster to cluster variations. The younger Alpha Persei cluster contains many more rapid rotators than the Pleiades and the largest rotational velocities are around $200 \mathrm{~km} / \mathrm{s}$ compared to around $100 \mathrm{~km} / \mathrm{s}$ in the low-mass Pleiades stars. While it is conceivable that this is due to the difference in the ages between the two clusters, it is also possible that the angular momentum distribution in all young clusters is not similar. Additional cluster data are required to investigate such issues.

\section{Evolution to the Main Sequence}

The problem gets more complicated when we try to understand the evolution of angular momentum from the pre-main sequence phase to the main sequence. While higher mass $\mathrm{T}$ Tauri stars show a large range in $v$ sini similar to that seen in early-type main sequence stars, lower mass $T$ Tauri stars show a surprisingly small range in $v \sin i$. The problem arises when the low mass $\mathrm{T}$ Tauri distribution is evolved to the main sequence (Bouvier 1991). To produce the fastest rotators seen in Alpha Persei, the most rapidly rotating T Tauri stars have to be spun up without any loss of angular momentum in the pre-main sequence phase. Such evolution will, however, not produce any of the slowly rotating stars seen in Alpha Persei and in IC 2391. Since the higher-mass T Tauris are at a later stage of evolution, their distribution may be an indication that the angular momentum loss is not uniform but depends on the velocity of the star. Pinsonneault (1992, these proceedings) suggests such a formulation. The alternative conclusion that $T$ Tauri stars are not representative of the pre-main sequence progenitors of stars in young clusters is unsubstantiated and premature but warrants further investigation (Bouvier 1991).

\section{From the Main Sequence to Giants}

When the early-type stars evolve off the main sequence and cross the Hertzsprung gap, their rotational velocities are observed to decrease. The drop in vsini is rapid as demonstrated by Gray (1989) and by the larger samples of de Medeiros and Mayor (1991) leading to the speculation that it is caused by the onset of the magnetic dynamo (see Gray 1991 for a review). In accordance with this scenario, Simon and Drake (1989) found that the chromospheric 
activity levels of giants and subgiants as measured in CIV and CII dropped across the Hertzsprung gap. Theoretical models have not yet demonstrated that such rapid braking is possible or investigated the effects on stellar composition.

\section{EFFECTS OF ROTATION}

One question raised as a result of our increased understanding of angular momentum evolution in stars is what is the effect of rapid rotation and spindown on the structure and interior of the star? In particular, does rotation induce mixing in the stellar interior and does it thus affect stellar composition? Theoretical models have postulated rotation-induced mixing by two principle means: meridional circulation as a result of rapid rotation and mixing as a result of spin-down. In this review, I will use the abundance of lithium to test if mixing has occurred. If mixing extends to the stellar surface we expect to see changes in the lithium abundances of main sequence stars. Mixing in the interior will not be revealed till the star becomes a subgiant or giant. Using lithium abundances to look for internal mixing is fraught with problems since we do not understand the lithium depletion mechanisms fully. For instance, lithium may be depleted as a result of turbulent diffusion or gravitational settling and these effects, if relevant, must be disentangled from rotation-induced mixing.

\section{Meridional Circulation}

Rapid rotation is expected to induce meridional circulation currents. Theoretical models (Charbonneau and Michaud 1988) have suggested that meridional circulation currents may be efficient enough to mix material up to the surface in early-F and late-A stars which have been rotating rapidly all their lives. Since lithium only survives in the outer $2-3 \%$ by mass of a typical main sequence star, mixing due to meridional circulation should dilute the surface abundance.

Unfortunately the equivalent width of the $\mathrm{Li}$ I resonance feature, the only measurable $\mathrm{Li}$ feature in most stars, is extremely small in these hot stars and not measurable in rapidly rotating stars. One way around this problem is to examine stars which have just evolved off the main sequence to slightly cooler temperatures. The surface abundances of these stars are not expected to have altered as a result of their evolution but their cooler temperatures make the $\mathrm{Li}$ I feature stronger. Such stars are not plentiful since, once they leave the main sequence, they evolve rapidly across the Hertzsprung gap, but the sample of $F$ stars observed by Balachandran (1990) provides a source. In that study, both lithium abundances and rotational velocities ( $v \sin i$ ' $s$ ) were measured for about 200 stars. Thirty six of these have $\mathrm{Mv}<2.4$ and have evolved from main sequence temperatures greater than $7500 \mathrm{~K}$. Since these stars are more massive 


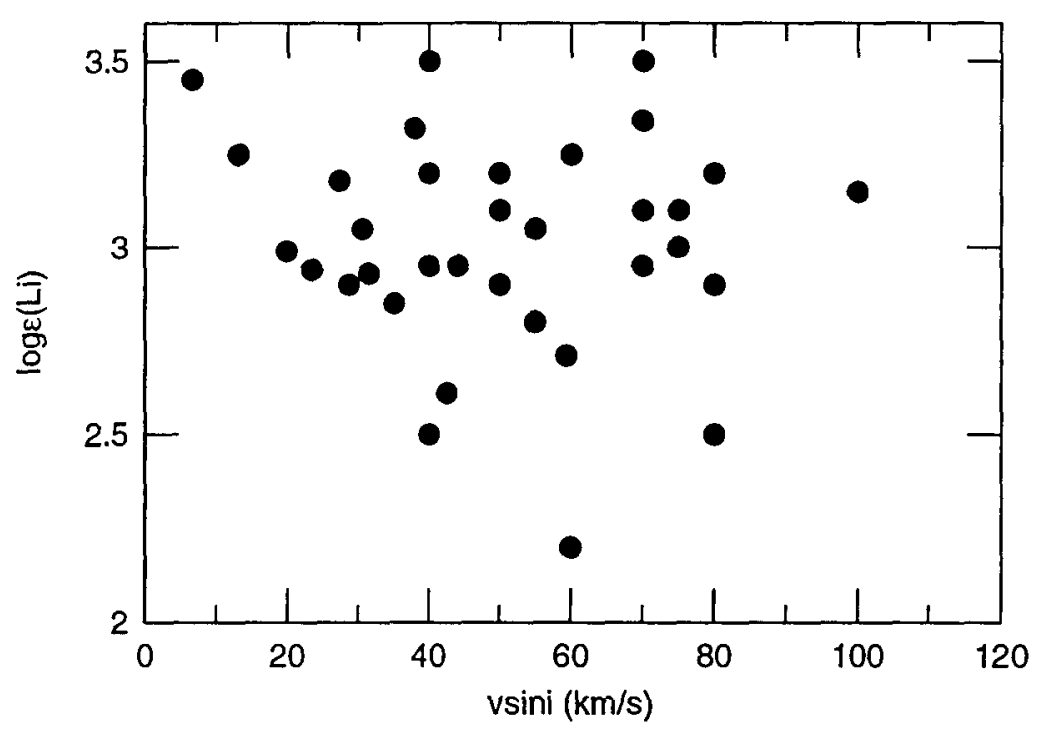

Fig. 1. Lithium abundances as a function of $v \sin i$ in $1.6-1.8 \mathrm{M}_{\odot}$ stars that have evolved from early-F and late- $A$ spectral types stars and are now seen crossing the Hertzsprung gap with temperatures between 6400K and 7000K (data from Balachandran 1990).

(1.6-1.8 $\left.\mathrm{M}_{\odot}\right)$ than lithium 'dip' stars $\left(\sim 1.35 \mathrm{M}_{\odot}\right)$ (Boesgaard and Tripicco 1986), their abundances will not be altered by the process affecting the 'dip' stars. In Figure 1, lithium abundances in these stars are plotted as a function of $v \sin i$. While the sample is small it contains stars rotating as rapidly as 100 $\mathrm{km} / \mathrm{s}$ and there is no evidence for a trend in lithium depletion with rotational velocity. At least in these massive stars, meridional circulation does not appear to have mixed material to the surface.

A similar conclusion was arrived at by Gilroy (1989) who examined the ${ }^{12} \mathrm{C} / 13 \mathrm{C}$ ratios in open cluster giants with ages between $50 \mathrm{Myr}$ and $5 \mathrm{Gyr}$ and found a large and constant ${ }^{12} \mathrm{C} /{ }^{13} \mathrm{C}$ ratio in giants more massive than $2.5 \mathrm{M}_{\odot}$ in accordance with standard dredge up theory. Clearly meridional circulation has not succeeded in mixing the ${ }^{13} \mathrm{C}$ peak outward in these stars. Smaller ${ }^{12} \mathrm{C} /{ }^{13} \mathrm{C}$ ratios were, in fact, seen in less massive stars (below $2.5 \mathrm{M}_{\odot}$ ) and the isotopic ratio was seen to decline with mass, reflecting perhaps the longer main sequence lives of these stars. The reason for the sharp change in ${ }^{12} \mathrm{C} /{ }^{13} \mathrm{C}$ ratio at this mass remains unknown. The observational evidence for meridional circulation related mixing is hence weak. 
Rotational Braking and Mixing

The first calculation of rotational braking in the Sun performed by Endal and Sofia (1981) predicted that mixing resulting from differential rotation may extend deep enough to destroy lithium. Since then more detailed models have been calculated by Pinsonneault et al. $(1989,1990)$. According to their models, when a star is braked on the main sequence, the outer convective zone spins down first leaving the interior rotating rapidly. This differential rotation causes turbulence which leads to mixing and lithium depletion. Their models parametrize the loss of angular momentum and introduce a factor to relate the transport of angular momentum to material transport so as to match the observed lithium depletion in the Sun. With these constraints, their models make quantitative predictions for the magnitude of lithium depletion in stars depending upon the initial angular momentum of the star and its age; the depletion increases both with the magnitude of the spin-down and with the age of the star. Since these are the only detailed models available for rotational braking, all observations will be compared to their predictions.

Unlike other lithium depletion mechanisms (e.g. turbulent diffusion) in which the depletion varies with age and spectral type alone, one of the major predictions of the Pinsonneault $e$ t al. (1990) model is that a spread in lithium will be observed at a given temperature in an old cluster, since these present-day slow rotators are expected to have had a range in initial angular mometum similar to that seen in young clusters. Lithium abundances have been measured in two old open clusters; M67 (Hobbs and Pilachowski 1986b; Garcia Lopez et al. 1988) and NGC 188 (Hobbs and Pilachowski 1988), and in one intermediate-aged cluster, NGC 752 (Hobbs and Pilachowski 1986a; Pilachowski and Hobbs 1988). The results are, at best, encouraging, at worst, inconclusive. In the oldest cluster, NGC 188, the scatter is not impressive. Five of the six stars measured have an average abundance of $\log \varepsilon(\mathrm{Li})=2.34 \pm$ 0.18 , a very small dispersion indeed. Only one star has a smaller abundance of $\log \varepsilon(\mathrm{Li})<1.8$. The observed sample in M67 is larger. A scatter of about 0.6 dex is seen in stars around the cluster turn-off at $6200 \mathrm{~K}$. Also, M67 stars, which are of the same age as the Sun, have larger than solar lithium abundances at solar temperature. In the sparsely-populated, intermediate-aged cluster NGC 752 three stars around solar effective temperature have a spread in lithium of 1.0 dex comparable to the spread predicted in the Pinsonneault $e t$ al. (1990) tabulations. However the lithium abundance drops sharply with temperature and some of the perceived scatter may be due to temperature uncertainties. Stronger evidence for a spread in lithium (though of smaller magnitude) is seen in the younger Hyades cluster long thought to have a tight Li-Teff relation. Recent observations by Thorburn et al. (1992) find a spread in lithium in stars around $6300 \mathrm{~K}$. Clearly, a large sample in a well-populated old cluster would 


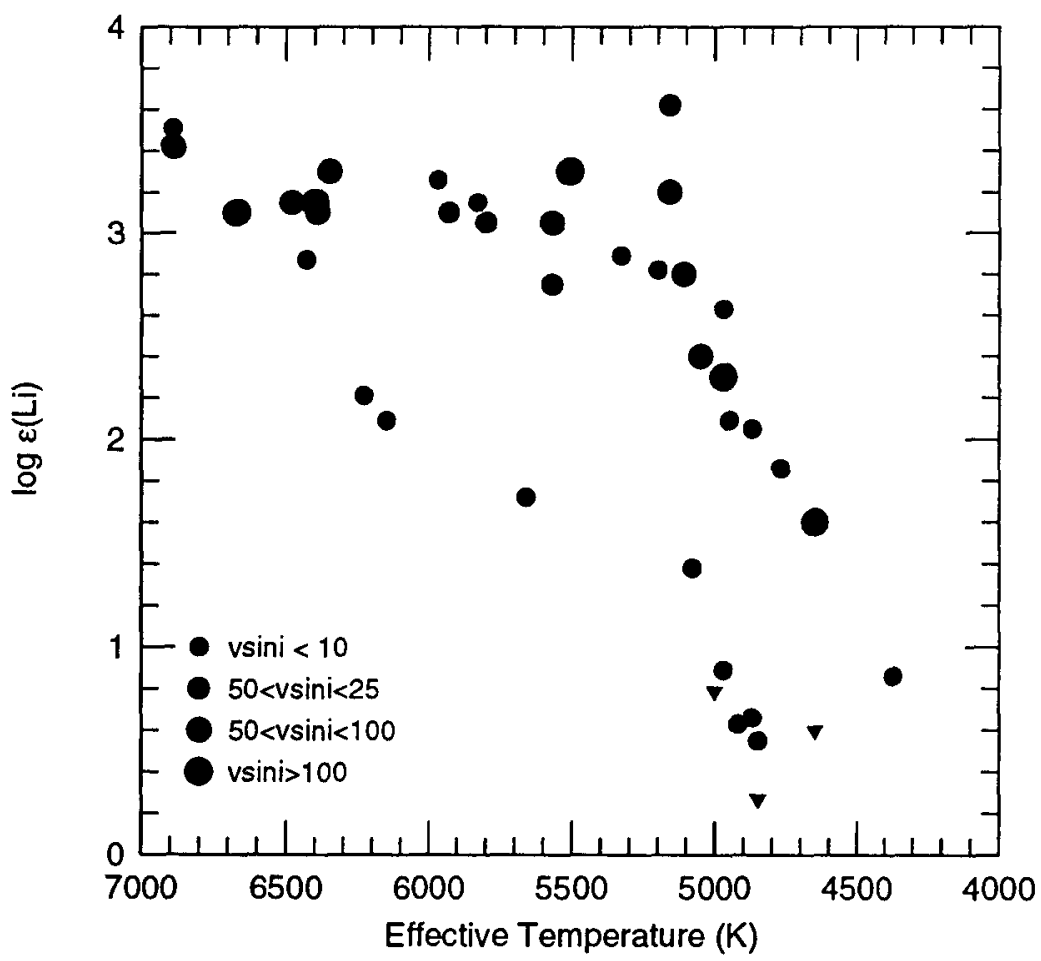

Figure 2. Lithium abundances as a function of effective temperature in the Alpha Persei cluster (from Balachandran, Lambert and Stauffer, 1988) with non-members from that sample discarded on the basis of new proper motion data from Prosser (1992). The stars are coded in $v \sin i$ as indicated in the key.

be of use. The basic question of whether rotational spin-down is capable of generating turbulence and material mixing is yet to be answered to the skeptics' satisfaction.

While the models do not predict a spread in lithium in young clusters, the observed samples are larger and the results are intriguing. In the Pleiades cluster (Soderblom et al. 1992, these proceedings), the abundances appear to be fairly well-behaved. With the exception of a few stars, the lithium abundances are a fairly smooth function of temperature with the hotter stars retaining their initial lithium abundances while the cooler stars reflect depletion expected to occur on the pre-main sequence. The abundance pattern in the younger Alpha Persei cluster is different. Using the new proper motion survey of Prosser 
(1992), non-member stars have been removed from our original study (Balachandran, Lambert and Stauffer 1988) and the remaining stars are plotted in Figure 2. The Li-Teff plot now exhibits two sequences of stars with the lower sequence populated exclusively by slow rotators though slow rotators are seen in the upper sequence as well. Since membership in the cluster is difficult to determine, skeptics may view any result from Alpha Persei as being clouded by the intrusion of non-members. It appears, however, that there are too many stars in the lower sequence to be accounted for by membership errors alone. The lithium abundance differences between the Pleiades and Alpha Persei, like the rotational velocity differences, may be pointing to a fundamental difference between the evolutionary history of the two clusters. If the lower lithium sequence is real (and additional data would be very useful to confirm this), Alpha Persei cannot evolve to look like the Pleiades. Whether the Alpha Persei and Pleiades lithium abundance patterns can evolve to the Hyades sequence is a very interesting and important question.

\section{Tidally-Locked Binaries}

Since the extent of lithium depletion in a star depends upon the magnitude of the spin-down in the Pinsoneeault et al. (1989) models, an interesting and testable consequence is that lithium depletion should cease when a binary star locks through tidal interaction. If tidal synchronization occurs early in the life of the star, i.e. the star is a short-period binary, then its lithium abundance would be anomalously high compared to other stars of the same age and effective temperature.

Soderblom et al. (1989) found larger than average lithium abundances in two short-period tidally-locked binaries in the Hyades, vB 22 and vB 62, and argued that the lack of spin-down in these stars may be responsible for their large abundances. However, Soderblom (1992) reported that KW 181 in Praesepe with a orbital period similar to $\mathrm{vB} 22$ has a lithium abundance below the average curve; the results in Pop. I stars are mixed.

Spite, Maillard and Spite (1986) reported a large lithium abundance in BD $0^{\circ} 4234$, a short-period binary with a metallicity of $[\mathrm{Fe} / \mathrm{H}]=-0.7$. An interesting and intriguing short-period, metal-poor binary is $\mathrm{HD}$ 89499. It has a circular orbit with a rotational period of 5.6 days (Ardeberg and Lindgren 1991). The secondary has not been detected. HD 89499 is particularly interesting because of both its age and its metallicity. According to Matheiu and Mazeh (1988), the orbit of such a short-period binary would have tidally-circularized around the age of the Hyades; tidal-synchronization would have occurred earlier. At that age, $\mathrm{Li}$ depletion would have ceased according to the Pinsonneault et al. (1990) models while a single star of the same temperature and metallicity would have continued to deplete Li. Since HD 89499 is old, the difference between its lithium abundance and a comparable single star will be large. Further, the 


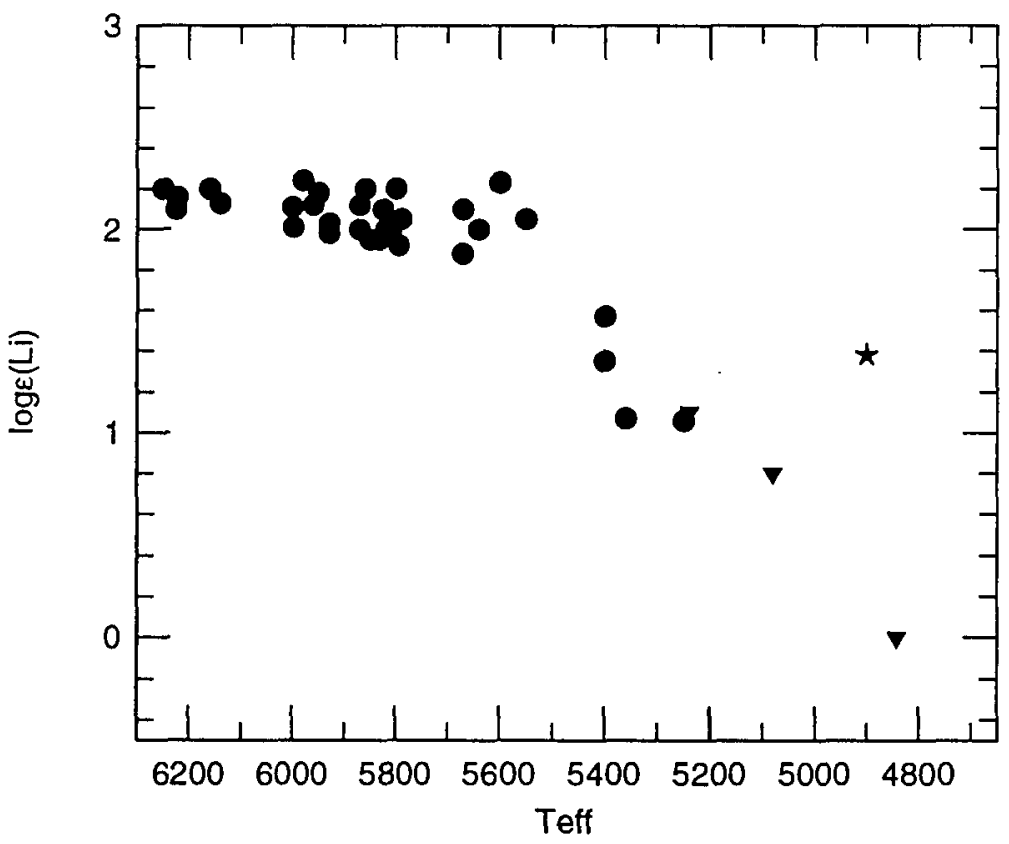

Figure 3. The lithium abundance in HD 89499 (star symbol) is shown in comparison with other stars on a lithium - effective temperature plot. The other stars are all metal-poor $([\mathrm{Fe} / \mathrm{H}] \leq-1.5)$ halo dwarfs. Effective temperatures and $\mathrm{Li}$ abundances for these stars are taken from Spite and Spite (1982, 1986), Spite et al. (1984), Hobbs and Ducan (1987), Rebolo et al. (1988) and Beckman et al. (1986). Filled circles are measured abundances and the inverted triangles are upper limits.

lithium abundances in Pop. II stars form a tightly defined sequence in the $\mathrm{Li}$ Teff plane. At temperatures greater than about $5300 \mathrm{~K}$, they form the lithium plateau first identified by Spite and Spite (1982). Observations are sparse at cooler temperatures but the overall scatter is much smaller than that seen in Pop. I stars. Recently Balachandran et al. (1992) observed the Li I feature in HD 89499. The abundance based on an effective temperature estimate of $4900 \mathrm{~K}$ is $\log \varepsilon(\mathrm{Li})=1.35$ which is a factor of 10 or higher than comparable single stars of the same-temeprature and metallicity (Fig. 3).

Some caution must be exercised before it can be concluded that the high $\mathrm{Li}$ abundance of $\mathrm{HD} 89499$ indicates a connection between $\mathrm{Li}$ depletion and rotational braking. The largest uncertainty in the interpretation of the $\mathrm{Li}$ abundance in $\mathrm{HD} 89499$ arises from the discrepancy between the vsini $(\sim 20$ 
$\mathrm{km} / \mathrm{s}$ ) measured from the spectrum and that derived from the rotational period. If the system is tidally locked, the rotational velocity of the dwarf $\mathrm{K}$ star must be around $6-8 \mathrm{~km} / \mathrm{s}$. The measured $v \sin i$ is consistent with the rotational period if the star has a larger radius, i.e. it is a subgiant. In this case its $\mathrm{Li}$ abundance simply reflects the diluted plateau value and is consistent with abundances seen in other halo subgiants (Pilachowski, Sneden and Booth 1992) thus having no bearing on the tidally-locked character of the system. It is hence crucial to the previous interpretation that this issue be resolved. The system, however, appears to be peculiar. Strömgren photometry places the star between the main sequence and subgiants. Further, examination of M92, a globular cluster of similar metallicity, shows that the subgiant branch is at much hotter temperatures. The possibility that the star may be heavily reddened and thus warmer is inconsistent with spectroscopically determined temperatures and in conflict with the Strömgren photometry calibrations of Schuster and Nissen (1989). This star is indeed a puzzle but other short-period binaries may hold the clue to the cause of $\mathrm{Li}$ depletion in stars. If tidal synchronization does indeed arrest lithium depletion, then a short-period binary on the Pop. II lithium plateau may reveal the value of the primordial lithium abundance.

\section{Chromospheric Activity. Rapid Rotation and High Li}

Near-cosmic lithium abundances have been observed in a few giants (Wallerstein and Sneden, 1982, Brown et al. 1989, Gratton and D'Antona 1989). The high $\mathrm{Li}$ abundances in these stars is puzzling because their low ${ }^{12} \mathrm{C} /{ }^{13} \mathrm{C}$ ratios indicate extensive mixing. A recent study of chromospherically active giants (Fekel and Balachandran 1992) sheds new light on these stars. The authors find that some of the chromospherically active giants are single; their rapid rotation is hence not a result of angular momentum derived from their orbits. Since observational data do not show any rapid rotators later than $\mathrm{G} 0$ on the subgiant branch, they conclude that these giants must have dredged up angular momentum from their rapidly rotating cores. A surprising find was the large lithium abundances in many of these stars (Fig. 4) leading to the suggestion that freshly synthesized lithium is being dredged up along with the angular momentum. Previously only AGB stars had been recognized as capable of producing lithium via the ${ }^{7} \mathrm{Be}$-transport mechanism in the hot-bottom convective envelopes of these stars (Smith and Lambert 1989; 1990). It has not been theoretically demonstrated that $\mathrm{Li}$-production is possible in first ascent giants but the observational evidence is intriguing enough to warrant further study. 


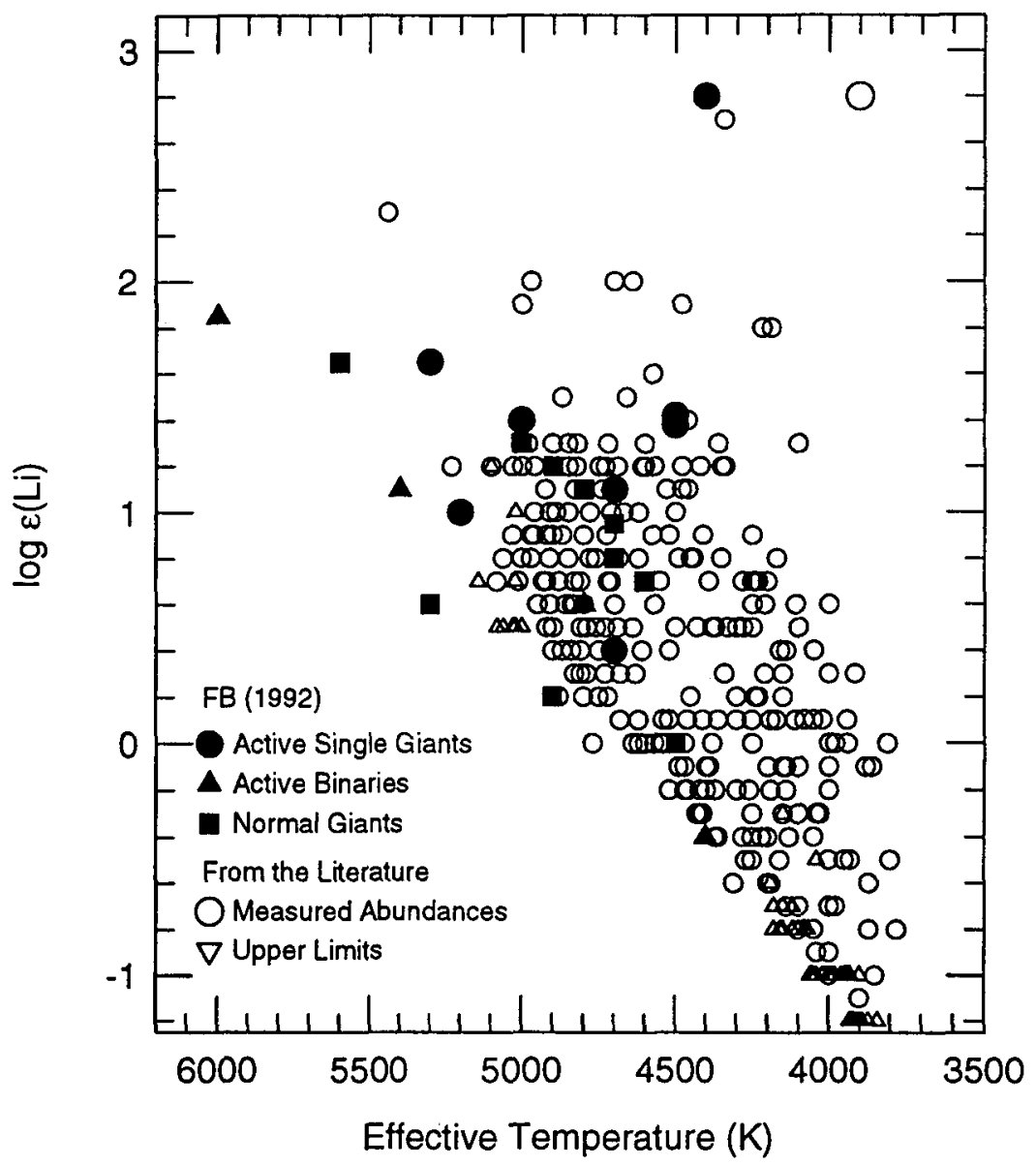

Figure 4. Lithium abundances versus effective temperature for giant stars. The filled symbols are from the sample of Fekel and Balachandran (1992). The remaining stars (open symbols) are from the sample of Brown et al. (1989) with the exception of two lithium-rich giants taken from Wallerstein and Sneden (1982) and Gratton and D'Antona (1989).

\section{SUMMARY}

We have gathered a considerable database on the rotational velocities in young stars. One major point that remains to be understood is the relationship between 
the rotational velocity distribution seen in young clusters and in pre-main sequence stars. We are now beginning to make inroads into understanding rotation-induced mixing. Based on theoretical models, it has been suggested that spin-down on the main sequence may be responsibe for considerable mixing and several observational tests for this model are possible. An obvious extension is to explain and understand the rotational slowing down of giants using a more rigorous theoretical framework. Conceivably such detailed understanding will lead to a more refined explanation for $\mathrm{Li}$-rich giants.

\section{Acknowledgements}

I would like to thank the American Astronomical Society for the International Travel Grant $A$ ward which enabled my attendance at this colloquium.

\section{REFERENCES}

Ardeberg, A. and Lindgren, H. 1991, A\&A, 244, 310.

Balachandran, S. 1990, ApJ, 354, 310.

Balachandran, S., Carney, B. W., Fullton, L. K., and Fry, A. M., 1992, BAAS, $24,767$.

Balachandran, S., Lambert, D. L. and Stauffer, J. R., 1988, ApJ, 333, 267.

Beckman, J. E., Rebolo, R., Molaro, P. 1986 in Advances in Nuclear Astrophysics, eds. E. Vangiori-Flam, J. Audouze, M. Casse, J. P. Chièze, and Tran Thanh Van, (Gif-sur-Yvette: Editions Frontières) p. 29.

Boesgaard, A. M. and Tripicco, M. J. 1986, ApJ, 302, L49.

Bouvier 1991, in Angular Momentum Evolution of Young Stars, eds. S.

Catalano and J. R. Stauffer, (Dordrecht: Kulwer).

Brown, J. A., Sneden, C., Lambert, D. L. and Dutchover, E. 1989, ApJS, 71, 293.

Charbonneau, P., and Michaud, G., 1988, ApJ, 334, 746.

de Medeiros, J. R., and Mayor, M. 1991, in Angular Momentum Evolution of

Young Stars, eds. S. Catalano and J. R. Stauffer, (Dordrecht: Kulwer).

Dearborn, D. S. P., Eggleton, P. P., and Schramm, D. N. 1976, ApJ, 203, 455.

Endal, A. J. and Sofia, S. 1981, ApJ, 243, 625.

Fekel, F. C. and Balachandran, S. 1992, ApJ, in press.

Garcia Lopez, R. J., Rebolo, R. and Beckman, J. E. 1988, PASP, 100, 1489.

Gilroy, K. K. 1989, ApJ, 347, 835.

Gratton, R. G. and D'Antona, F. 1989, A\&A, 215, 66.

Gray, D. F. 1989, ApJ, 347, 1021. 
Gray 1991, in Angular Momentum Evolution of Young Stars, ed. S. Catalano and J.R. Stauffer, (Dordrecht: Kulwer).

Hobbs, L. M. and Duncan, D. K. 1987, ApJ, 317, 796.

Hobbs, L. M. and Pilachowski, C. 1986a, ApJ, 309, L17.

Hobbs, L. M. and Pilachowski, C. 1986b, ApJ, 311, L37.

Hobbs, L. M. and Pilachowski, C. 1988, ApJ, 334, 734.

Kraft, R. 1970, in Spectroscopic Astrophysics, ed. G. H. Herbig (Berkeley:

University of California press), p. 385.

Lambert, D. L., and Ries, L. M. 1977, ApJ, 217, 508.

Mathieu, R. D. and Mazeh, T. 1988, ApJ, 326, 256.

Pilachowski, C. and Hobbs, L. M. 1988, PASP, 100, 336.

Pilachowski, C. A., Sneden, C., and Booth, J. 1992, preprint.

Pinsonneault, M. H., 1992, in Inside The Stars, eds. W.W. Weiss and A.

Baglin, in press.

Pinsonneault, M. H., Kawaler, S. D., and Demarque, P. 1990, ApJS, 74, 501.

Pinsonneault, M. H., Kawaler, S. D., Sofia, S., and Demarque, P. 1989, ApJ, $338,424$.

Prosser. C. F. 1992, AJ, 103, 488.

Radick, R. R., Thompson, D. J., Lockwood, G. W., Duncan, D. K., and

Baggett, W. E. 1987, ApJ, 321, 459.

Rebolo, R., Beckman, J. and Molaro, P. A\&A, 172, L17.

Scalo, J. M., and Miller, G. E. 1978, ApJ, 225, 523.

Schuster, W. J. and Nissen, P. E. 1989, A\&A, 221, 65.

Simon, T. and Drake, S. A. 1989, ApJ, 346, 303.

Smith, V. V. and Lambert, D. L. 1989, ApJ, 345, L75.

Smith, V. V. and Lambert, D. L. 1990, ApJ, 361, L69.

Soderblom, D. R., Oey, M. S., Johnson, D. R. H., and Stone, R. P. S. 1990, AJ, 99, 595.

Soderblom, D. R., Stauffer, J. R., Jones, B. F. and MacGregor, K. 1992, in Inside The Stars, eds. W.W. Weiss and A. Baglin, in press.

Spite, M., Maillard, J. P., and Spite, F. 1984, A\&A, 141, 56.

Spite, F. and Spite, M. 1982, A\&A, 115, 357.

Spite, F. and Spite, M. 1986, A\&A, 163, 140.

Stauffer, J.R. 1991, in Angular Momentum Evolution of Young Stars, ed. S.

Catalano and J.R. Stauffer, (Dordrecht: Kulwer)

Stauffer, J., and Hartmann, L. 1987, ApJ, 318, 337.

Stauffer, J., Hartmann L., and Jones, B. 1985, ApJ, 289, 247.

Stauffer, J., Hartmann L., and Jones, B. 1989, ApJ, 346, 160.

Stauffer, J., Hartmann, L. W., Jones, B. F., and McNamara, B. R. 1989, ApJ, 342, 285.

Stauffer, J., Hartmann, L., and Latham, D. 1987, ApJ, 320, L51. 
Thorburn, J. A., Hobbs, L. M., Deliyanis, C. P. and Pinsonneault, M. H. 1992, Ap.J., submitted.

van Leeuwen, F. and Alphenaar, P. 1982, ESO Messenger, No. 18, p. 15.

Wallerstein, G. and Sneden, C. 1982, ApJ, 255, 577. 